\title{
Elites de izquierda: experiencias de vida y relatos autobiográficos para comprender la transición en Chile ${ }^{*}$
}

Cristina Andrea Moyano Barahona**

Resumen: Artículo que aborda la configuración, reproducción y mantención de las elites chilenas de izquierda durante la dictadura, poniendo especial énfasis en el enfoque de redes y en particular, en el análisis de las trayectorias biográficas de un conjunto de líderes, con el fin de comprender no sólo las prácticas de dicho grupo, sino que también la configuración de una cultura política que será clave para analizar el proceso de transición a la democracia en Chile.

Palabras claves: Elites de izquierda. Redes sociales. Trayectorias autobiográficas militantes.

\section{Introducción}

El estudio de la izquierda durante el período dictatorial conlleva un sinnúmero de problemas para su análisis historiográfico. En primer lugar implica sumergirse en un período cuya excepcionalidad genera patrones de comportamientos nuevos y adecuados para la situación. Clandestinidad, exilio, represión, miedos, etc., nos abren nuevos escenarios analíticos para acercarnos a estos sujetos, con los problemas adicionales referidos a la escasez de fuentes oficiales, dispersión de las mismas, lo que nos lleva inevitablemente a recuperar experiencias por medio de las entrevistas en profundidad.

\footnotetext{
* Proyecto Fondecyt Regular 1120009

${ }^{*}$ Doutora em História - Universidad de Santiago de Chile. E-mail: cristina. moyano@usach.cl.
}

Anos 90, Porto Alegre, v. 20, n. 38, p. 385-410, dez. 2013 
Elites de izquierda: experiencias de vida y relatos autobiográficos...

En el marco del desarrollo del proyecto Fondecyt-Posdoctoral No 3085033 y del nuevo proyecto Fondecyt Regular 1120009, nos propusimos investigar las redes sociales que permitieron la subsistencia de la izquierda y en particular, la configuración de los liderazgos político-partidarios que articularon la elite de este sector. Habiendo recurrido a distintas fuentes previas para identificar a los líderes políticos y los mecanismos de validación y legitimación de los mismos, nos centraremos ahora en el análisis de las entrevistas en profundidad, para ampliar y concentrarnos en las redes sociales que pueden extraerse de los relatos.

La hipótesis central de este texto puede dividirse en tres partes. En primer lugar afirmamos que las condiciones tradicionales en las que la izquierda había constituido a sus líderes y su legitimación se quiebran con la instalación de la dictadura, lo que genera nuevas condiciones y la necesidad del surgimiento de nuevas prácticas para constituir nuevos liderazgos, especialmente, cuando muchos líderes fueron muertos, desaparecidos, exiliados y apresados por el nuevo régimen político. En ese nuevo espacio, se necesitó repensar los mecanismos de generación de liderazgos y de validación de los mismos. Ante la ausencia de la posibilidad de realizar elecciones, trabajo de masas, etc., la izquierda hubo de redefinir sus prácticas y esto generó nuevas formas de constitución de liderazgos, basados mayoritariamente en la cooptación de militantes que reunieran ciertas características apropiadas para la nueva situación, especialmente en el interior del país.

En segundo lugar, nuestra hipótesis de trabajo apunta a señalar que estos mecanismos de cooptación se establecieron mayoritariamente sobre redes sociales que tenían los militantes políticos, lo que genera la necesidad de estudiar a las mismas para llegar a comprender las razones de la emergencia de nuevos liderazgos y por sobre todo, las conexiones internas y externas dentro de los partidos de izquierda, que posibilitaron alianzas, contactos y circulación de ideas, representaciones e imaginarios.

En tercer lugar, estas dinámicas de cooptación como forma de reproducción de la elite de izquierda se fueron transformando en una práctica cultural que se convirtió en uno de los lastres más significativos para los procesos de redemocratización de los parti- 
dos durante la transición a la democracia y que explica, en parte, las dinámicas de distanciamiento de los liderazgos políticos con la ciudadanía y los movimientos sociales, convirtiéndose en el soporte de una cultura política transicional excluyente, y que generó una estructura de reproducción de las elites basada más en la integración horizontal que en la vertical. Se suma a lo anterior, la valoración de ciertos tipos de liderazgos, donde la figura del "técnico político", cobró mayor relevancia, fortaleciendo una visión técnica del proceso político, donde las redes con los actores sociales se estructuraron en base a la formalidad de las relaciones de cooptación por medio de los "operadores políticos" y el diseño de políticas públicas que permitieron canalizar las demandas sin la participación directa de los actores sociales. Este último proceso se acrecienta en el período de Eduardo Frei Ruiz Tagle, pero su constitución inicial viene en la izquierda desde los años 80 (MOYANO, 2009).

En esa perspectiva hemos recurrido a los relatos biográficos de las trayectorias militantes de quienes han sido identificados como principales líderes epocales de la izquierda para el período 1973-1989. Sus entrevistas nos han proporcionado elementos claves para comprender las conexiones y redes, así como las formas en las cuáles la propia izquierda articuló nuevos liderazgos políticos, articulando una nueva elite que convivió tensionadamente con los viejos sobrevivientes de la dictadura, que comenzaron a regresar a nuestro país a mediados de los años 80 .

\section{La entrevista y la trayectoria militante}

Una trayectoria militante corresponde a las vivencias, experiencias, acciones y construcciones de redes sociales relacionales, posiciones y ocupaciones que se constituyen en la práctica militante de los sujetos. En ese sentido una trayectoria militante tiene como componentes las vicisitudes que los actores sostienen mientras militan, aunque el inicio de dicha actividad esté marcada por distintos elementos claves como el capital social del que dispone al inicio de la militancia, características personales de liderazgos y las propias condiciones contextuales en las cuáles se estructuran las practicas. 
Elites de izquierda: experiencias de vida y relatos autobiográficos...

En esa perspectiva la actividad militante, entendida como praxis cotidiana en función de las ideas e imaginarios que estructura la comunidad partidaria, va generando nuevas formas relacionales, nuevas vinculaciones interpares, que van constituyendo "camarillas" internas, que estructuran espacios de apoyo, de acumulación de capital social y que en ciertos momentos particulares pueden convertirse en claves para asumir, legitimar o validar liderazgos partidarios.

En ese sentido, las entrevistas biográficas nos permiten determinar elementos referidos al capital social inicial, a las redes sociales que constituyen los militantes en la militancia activa y cómo inciden las mismas en la validación, legitimación de nuevos liderazgos, así como en las decisiones en las que se combina la reflexión analítica y la subjetividad afectiva. Sin embargo, pese a permitir una transferencia de realidad, es importante considerar los propios límites devenidos de cualquier relato generado desde un presente específico.

La consideración de que los sujetos hablan desde un presente no enunciado, es una clave para comprender las formas de trabajo de la memoria. Sin embargo, pese a los cuidados que este tipo de fuentes contiene, su pertinencia metodológica en este trabajo es invaluable. En los relatos de los militantes, en el decurso de la enunciación de su trayectoria militante los sujetos van enunciando a los otros con quienes compartieron sus experiencias de vida, sujetos con los cuales van articulando una red relacional que aparece en sus relatos de manera precisa, conteniendo datos respecto de situaciones contextuales, grados de amistad, confianza, posiciones de los otros, distancias sociales (MOYANO, 2010), etc.

Los recuerdos van configurando de esta forma la propia red del sujeto en años particulares, en los cuales probablemente queden varios sujetos fuera de los mismos, producto de las inexactitudes y olvidos del trabajo de la memoria. En entrevistas comparadas de militantes de mismas tiendas políticas, se van dibujando los líderes más influyentes, quienes son nombrados como claves para ciertos periodos. Aparecen también los líderes olvidados, aquellos que en el presente pueden parecer como desconocidos y que requieren de atención especial para comunicar al investigador sus características contextuales a destacar. En esa perspectiva, el análisis de dichos 
relatos nos permite dibujar ciertas redes sociales de los líderes de la izquierda en dictadura, considerando los siguientes aspectos teórico-metodológicos provenientes del enfoque de redes.

\section{Enfoque de redes}

El enfoque de redes parte de una premisa básica y es que los sujetos no son átomos indivisibles, sino que un conjunto coherente de relaciones " $[. .$.$] tanto físicas como intelectuales, con la$ naturaleza, con los objetos, con las otras personas, relaciones que la transforman continuamente. Así ningún individuo es estrictamente individual (VILLASANTE, <http:/ / www.ucm.es/info/pecar/Articulos/Villasante21.pdf $>$ )", sino que un ser inserto en relaciones sociales. Esas relaciones sociales permiten hacer circular las ideas, generan confianzas, posibilidades de agrupación, construcción de imaginarios, que en la historia política reciente son claves para comprender las decisiones de colectividades respecto de alianzas, ideas y reflexiones, que no siempre pueden explicarse desde una perspectiva meramente de transformación ideológica racional y aislada de esas relaciones que las sostienen. De esta forma la coherencia de estas relaciones puede cambiar en el tiempo, reforzarse, consolidarse o destruirse y eso debe ser estudiado históricamente.

Para Tomás Villasante

[...] nuestros comportamientos como soportes de relaciones sociales dependen de cómo funcionan las redes en las que nos encontramos en cada caso. Y, las reglas de comportamiento de cada red es algo que debemos estudiar por la etnología de las sociedades complejas. Sin duda cada red está influenciada decisivamente por los condicionantes económico-políticos y por los espacios en que vive. Pero el soporte simbólico de la red en numerosas ocasiones aparece en primer plano para mostrar que los inconscientes individuales e históricos tienen un peso muy importante. Los soportes individuales, familiares o grupales tienen tanto de comportamientos racionalizados de acuerdo con sus necesidades objetivas, como de elementos de la cultura familiar, local o social. El soporte es 
Elites de izquierda: experiencias de vida y relatos autobiográficos...

sexo y género, es también necesidades primarias y culturales, es en suma la historia de muchas redes que se entrelazan en una sociedad compleja (VILLASANTE, 2006, p. 14)

perspectiva que funciona adecuadamente para el análisis de las culturas políticas partidarias, porque la cotidianeidad de la militancia va generando lazos y visiones de mundo compartidas, no sólo en relación con los mismos militantes de la propia colectividad, sino que en relación con los partidos con los cuales se estructuran relaciones de alianza, convivencia y confianza.

Un análisis de redes puede realizarse considerando dos enfoques que pueden resultar complementarios. En primer lugar el enfoque que centra su atención en la búsqueda de cohesión, es decir, que se orienta a determinar las características de los lazos relacionales que se establecen entre dos o más sujetos. Del análisis de los lazos relacionales se pueden obtener las medidas de rango ${ }^{1}$, grado de intermediación ${ }^{2}$, cercanía ${ }^{3}$ y densidad ${ }^{4}$.

Un segundo enfoque es aquel que pone más atención en las posiciones que ocupan los sujetos dentro de una red, es decir, centra su análisis en los lugares en los que se encuentran los sujetos respecto de los otros y los tipos de conexiones que establecen entre ellos. Este enfoque, complementario al anterior, también es clave para analizar las culturas políticas partidarias y las elites de la izquierda, ya que nos permite determinar sujetos influyentes, con prestigio e importantes al interior de las colectividades o como sujetos que ejercen la función de transversalidad entre las mismas.

La actividad política y por sobre todo, la actividad militante, constituye una praxis donde la construcción de redes es inherente. La posibilidad del cambio social, base del discurso político de la izquierda, depende de la construcción de lazos entre los que pertenecen a la misma red militante y los diferentes, es decir, aquellos con los que se puede realizar alianzas políticas circunstanciales y de largo plazo. En esa perspectiva la teoría de los lazos débiles, pero influyentes, de Granovetter, también constituye un insumo para analizar las trayectorias políticas militantes, los éxitos de las alianzas y las propias posibilidades de acción de los sujetos.

Así, las experiencias militantes que estructuran bases aglutinantes de las memorias de los sujetos, nodos articuladores de re- 
flexiones analíticas sobre su propio comportamiento político, nos permiten acercarnos a las conexiones de estos en perspectiva histórica. Como plantea Villasante, para ese análisis

[...] lo mejor es observar o preguntar por un acontecimiento concreto, suficientemente conocido por la mayoría (un "analizador"). Hay hechos en cualquier colectividad que influyen decisivamente en el posicionamiento de las redes: este "analizador" sería como el "ego" ordenador para los antropólogos de redes. Este suceso/analizador, al ser algo que rompió los hábitos cotidianos, y obligó a posicionarse en la red de distintas formas a cada posición, viene a contrastar con los habituales comportamientos, por lo que también nos muestra tendencias pasadas y contrastes (VILLASANTE, <http://www.ucm.es/info/pecar/Articulos/Villasante21.pdf> ).

De esta forma, considerar estos elementos como claves al momento de analizar los relatos biográficos puede complementar los análisis tradicionales en el plano de las elites políticas, en especial en la historiografía.

$\mathrm{Al}$ respecto proponemos el siguiente esquema analítico para abordar las redes sociales de las trayectorias militantes, que operativizaremos con una pequeña muestra de entrevistas de militantes políticos destacados.

- Partido Político al que pertenece.

- Capital cultural de entrada: origen familiar/vinculación previa con la política/ colegio/ universidad/profesión.

- Hitos claves de la vida y sujetos con que los vivencia.

- Actividades políticas relevantes y sujetos con que los vivencia.

- Reconocimiento de líderes claves en su trayectoria política (relaciones verticales de admiración/influencia)

- Reconocimiento de pares con los que ha construido relaciones de mayor confianza.

- Reconocimiento de pares de otras colectividades con los que haya construido relaciones de mayor confianza.

- Estos siete puntos nos permiten extraer los siguientes elementos:

- Densidad de las redes.

- Centralidad de liderazgos.

- Distancia.

- Conexiones.

Anos 90, Porto Alegre, v. 20, n. 38, p. 385-410, dez. 2013 
Elites de izquierda: experiencias de vida y relatos autobiográficos...

- Puentes.

- Caracterización de trayectorias militantes y culturas políticas de la izquierda en dictadura.

\section{La vieja izquierda: reproducción y selección}

Dentro de la vieja izquierda consideraremos los casos referidos al Partido Socialista de Chile y al Partido Comunista (ALVAREZ, 2011). Ambas colectividades poseen una trayectoria de casi medio siglo al momento del golpe de Estado, cuya historia configura una cultura política particular y una identidad forjada a la luz de variadas experiencias históricas, en las que se combinaron experiencias gubernamentales de colaboración, de clandestinidad y de resistencia.

En ese marco existía en ambas colectividades diversos tipos de liderazgos. En el Partido Comunista por ejemplo, las trayectorias pre 1973 estaban marcadas por la militancia social que se convertía en espacio clave desde donde se construía el capital político. $\mathrm{Al}$ respecto son muy interesantes las trayectorias biográficas de Luis Corvalán Lepe, para citar un caso gráfico. Sin embargo, hacia la década de 1960 también comienzan a configurarse como nuevos espacios de constitución de capital político, las experiencias universitarias que se vuelven relevantes para la emergencia de nuevos líderes políticos. Estos son los casos de Jorge Insunza $a^{5}$ de José Cademartori ${ }^{6}$, por ejemplo, así como el de Patricio Palma y de Manuel Fernando Contreras ${ }^{8}$.

El espacio universitario hacia la década de los 60 se convirtió en un importante espacio de reclutamiento de líderes jóvenes, que no proviniendo de familias vinculadas a la actividad política más tradicional, comienzan a leer la praxis política vinculada con el pensar y actuar en función de su preparación profesional. En este ámbito de desarrollo militante, los nuevos reclutados ocupan rápidamente espacios de poder vinculándose a la actividad universitaria e insertándose en actividades partidarias de propaganda, difusión y nuevos reclutamientos.

Es importante destacar también, que la posibilidad de movilidad en ascenso de los nuevos militantes estuvo vinculada a la 
cercanía a ciertos líderes, miembros de las respectivas elites de sus partidos, que hacían la vez de selectores y entregaban legitimidad a la nueva carrera militante. Esta vinculación permitía un proceso de reproducción de las elites, que si bien era lento, tiende a acelerarse con la incorporación de numerosos cuadros jóvenes en los años 60 y 70, previo golpe de Estado, llegando a su punto culmine con el gobierno dirigido por Salvador Allende.

Los líderes claves que cumplieron ese rol de selectores en el PC de Chile fueron, según el cuadro de entrevistas realizado en el marco de esta investigación, las figuras de Luis Corvalán Lepe, Orlando Millas y Galo González, todos miembros de la dirección del Partido, miembros de Comisiones Políticas y en dos de dichos casos, Secretarios Generales por extensos períodos de tiempo.

De esta forma, el PCch combinaba la selección por la vía de la cooptación de los viejos líderes que ejercían el rol de institucionalización de legitimidad política, permitiendo ascensos estructurales dentro de la colectividad, así como la configuración de trayectorias políticas sociales dilatadas en el marco de frentes sociales de masas que dotaban al militante de una validación sociopolítica básica para su legitimidad partidaria.

Para el caso del Partido Socialista de Chile, dada la cultura política fraccionalista, es posible destacar una situación relativamente similar a la descrita previamente para el PCch. Los viejos líderes socialistas tenían un capital social vinculado a la militancia social y a la participación en los años 30 y 50 en distintas reparticiones gubernamentales. A diferencia del PC., el Partido Socialista combinaba con mayor naturalidad a jóvenes provenientes de estratos medios y medios acomodados con el mundo de los trabajadores. De allí que la militancia juvenil que se integra en los años 60 no transforme significativamente la configuración social de la colectividad.

Sin embargo, en el Partido Socialista la cercanía a ciertos líderes claves es muy importante para garantizar el acceso a una trayectoria política fecunda. En ese sentido, la cultura fraccionalista y caudillista presente en los viejos liderazgos socialistas, se convierte en una clave para comprender los procesos de reproducción y legitimación de los cuadros integrados a la colectividad. A diferencia del partido Comunista, donde la cercanía es una fuente de 
Elites de izquierda: experiencias de vida y relatos autobiográficos...

legitimidad político institucional, en el Partido Socialista es uno de los principales focos productores de capital político, siendo la cooptación por la vía de los viejos liderazgos la principal forma de ascenso y de integración a la elite del partido.

En ese sentido, jugaron un rol clave en la selección de líderes dentro del Partido Socialista, las figuras de Salvador Allende y de Clodomiro Almeyda, significados en las entrevistas como líderes relevantes en el reclutamiento político. Es importante señalar a su vez, que la importancia de estos dos actores es significativa no sólo para el mundo socialista, sino que excede con creces a la mayoría de la izquierda, tanto la nueva como la vieja.

Es interesante que de los líderes socialistas entrevistados, un porcentaje significativo de las entrevistas (más del 90\%), correspondan a líderes cuyo espacio de reclutamiento fue el ámbito universitario. Desde ese ámbito de formación profesional y de práctica política se produce un buen puente de nutrición entre la formación de los cuadros políticos y las elites de las distintas directivas socialistas. Así es significativo por ejemplo, mencionar el rol que jugó en esa área, Clodomiro Almeyda.

Clodomiro Almeyda ejerció como profesor universitario y desde ese cargo logró reclutar a numerosos dirigentes políticos, quienes como ayudantes de cátedra o de investigación, estuvieron formativamente cerca de dicho personaje político. Más tarde, Clodomiro Almeyda convocaba a dichos ex discípulos para integrarlos a la administración del Estado entre 1970 y 1973. De esta forma, logró extender vastas y densas redes entre el mundo socialista joven que asume la reconstrucción del partido en clandestinidad, así como con liderazgos de la nueva izquierda, en especial, con ciertos militantes del MAPU.

Por su parte, existen otros líderes socialistas que son mencionados en las entrevistas como "influyentes", pero cuya influencia no puede ser medida de manera tan categórica como la del propio Almeyda, y se reduce a una influencia simbólica, intelectual o política, sin que ello implique el desarrollo de redes sociales amplias y densas en donde estos personajes se conecten.

$Y$ cuando se derrumba la democracia.

La situación antes descrita, tanto al interior del PCch como en el PSch, sufre modificaciones cuando acontece el golpe de Es- 
tado. $\mathrm{Al}$ respecto, podemos inferir de las entrevistas realizadas, que una vez que se desata la represión política y las condiciones de clandestinidad se vuelven las hegemónicas, las distintas colectividades comienzan a rearticular sus estrategias de sobrevivencia, dentro de las cuales una de las más significativas era la reestructuración de las directivas políticas tanto en el interior como en el exterior.

El mundo comunista y socialista logró salvaguardar conflictivamente, y con muchas pérdidas, a los líderes más visibles de sus conglomerados, siendo sus cuadros dirigenciales de segunda línea los más afectados por la represión. En ese sentido, la necesidad de reestructurar una línea dirigencial en el interior se volvió relevante para mantener una militancia política que pudiera enfrentar a la dictadura.

La mayoría de los cuadros que asumen esa labor en Chile fueron cuadros jóvenes, especialmente para el período 1973-1978, cuya legitimidad política para asumir dichas tareas provenía de los siguientes espacios de poder:

- Pertenencia previa a la Comisión Política, ya sea del Partido o a la Juventud del mismo.

- Pertenencia al Comité Central del Partido en calidad de miembro titular o suplente del mismo.

- Cercanía personal/conocimiento previo a algún miembro del Comité Central que posibilita la integración por la vía de diversas labores militantes.

El último de los puntos señalados nos parece el más relevante, porque imprime un sello nuevo al periodo dictatorial, respecto de la selección y reproducción de liderazgos en estas colectividades. Si bien es difícil medir la cercanía personal de los líderes políticos emergentes respecto de los viejos líderes instituidos, y con validación dentro de la estructura interna de la colectividad, es importante señalar que aquí cobra especial relevancia las redes sociales de quienes asumen la reconstrucción partidaria en ambas colectividades.

Así es posible señalar que en el mundo socialista sobresalen para el período que se extiende entre 1973 y 1978 los siguientes nuevos tipos de líderes políticos emergentes, reclutados por la vía de la cooptación arbitraria y contextual de los viejos liderazgos, depositarios en alguna legitimidad institucional: 
Elites de izquierda: experiencias de vida y relatos autobiográficos...

Tipos de liderazgos emergentes, instituidos por la vía de la cooptación política:

- Liderazgos intelectuales que ejercen un rol no partidario, de manera directa, pero que cumplen funciones de enlace, de mantención de una "fachada" o manto y que permiten la circulación de reflexiones políticas tanto a nivel partidario como a nivel público (Ejemplo: Ricardo Lagos ${ }^{9}$, Angel Flisfisch $^{10}$, Jorge Arrate ${ }^{11}$, entre otros).

- Liderazgos partidarios internos cuyas características personales les permitieran realizar trabajos autónomos y mantener una vida no "sospechosa" de las autoridades militares vigentes. En ese sentido es muy importante analizar las características de su propio capital social. (Ejemplo: German Correa ${ }^{12}$, Hernán Vodanovic ${ }^{13}$, Ricardo Nuñez $\left.{ }^{14}\right)$.

- Liderazgos partidarios internos cuyas experiencias pasadas los validaran como sujetos confiables, dadas las circunstancias políticas nuevas instauradas con el golpe de Estado. (Ejemplo: Ricardo Solari ${ }^{15}$ )

Estos tipo de liderazgos presentan conexiones con los antiguos líderes y se caracterizan por asumir (caso 2 y 3) funciones políticas en un ambiente hostil y peligroso. A ellos se les debe la reconstrucción y mantención orgánica de la colectividad al interior del país. Así, no teniendo trayectorias políticas dilatadas, las circunstancias y su capital social, les permiten construir un capital político significativo dentro de la estructura política interna de la colectividad.

El período que se abre posterior a 1979, abre en el PS, después de su división, un espacio para la existencia de otros reclutamientos, todavía basados en la cooptación, pero con una mayor vinculación con la práctica política militante más pública. Así, el surgimiento de espacios de discusión política en las universidades, en los sindicatos y en otras organizaciones sociales en ciernes, permitió la incorporación de nuevos líderes cuya integración fue en muchos casos conflictiva, tal como muestran los relatos biográficos con los que trabajamos.

La práctica política militante en clandestinidad comienza, hacia los inicios de la década de los 80 , a tensionarse con la práctica política más abierta y pública. La validación y legitimidad de los militantes emergentes en clave de "liderazgo", se conflictua con las validaciones por la vía de la cooptación y comienza a generar 
tensiones por la reproducción e integración de los nuevos cuadros políticos. Dos experiencias ${ }^{16}$ políticas distintas, generan dos modos de percibir, enunciar y reflexionar la actividad política, en ese marco, la cooptación comienza a cuestionarse y se pone en el tapete de la discusión la validación por la vía de la trayectoria, de la obra militante y no por la cercanía o el reclutamiento de los viejos líderes políticos, que mayoritariamente en el exilio, también comienzan a ser duramente cuestionados.

En este nuevo escenario emergen líderes, cuya capacidad de incorporación estará mucho más vinculada a su capacidad para relacionarse con los viejos liderazgos, es decir, para establecer puentes de conexión con el viejo mundo político, con la elite consolidada por la práctica de la clandestinidad y el exilio. En ese sentido, podemos afirmar que estos nuevos liderazgos, emergentes en el seno de las luchas de resistencia en las poblaciones, en las universidades, en frente sociales, entre otros, asumirán también su propia validación por la vía de una cooptación menos fuerte, cuya fuente de legitimidad sigue respetando la vieja institucionalidad partidaria. De allí que su mundo de la vida se subsuma al mundo de la vida de los reconstructores del partido.

Por su parte, el caso del PCch, dada la estructura jerárquica y disciplinada que destacan sus propios militantes en las distintas entrevistas realizadas, nos permite concluir que la cooptación por la vía del reclutamiento de cuadros políticos no tuvo grandes conflictos internos. Las validaciones provenían de los viejos líderes de la colectividad, que una vez en el exilio o en el interior del país, van dotando de legitimidad a los nuevos miembros que van asumiendo funciones directivas.

Los mayores conflictos que evidencia el PCch ocurren hacia la segunda mitad de la década de los 80 y está en el marco de dos procesos concomitantes. En primer lugar, el distanciamiento que un sector de militantes hace del PC después del fracaso de la vía insurreccional, denominada Rebelión Popular de Masas. El distanciamiento proviene tanto de los cuadros militares que forman parte de la dirección del FPMR, para quienes el abandono de esta política era un error político significativo y que los dejaba sin poder político dentro de la colectividad; así como también de aquellos 
Elites de izquierda: experiencias de vida y relatos autobiográficos...

otros liderazgos, que ejerciendo labores más públicas, tanto en actividades sociales como en las organizaciones políticas como el Movimiento Democrático Popular (MDP) o más tarde Izquierda Unida (IU), van compartiendo espacios de mundo de la vida que los van distanciando de las experiencias de los cuadros dirigenciales que mantenían su clandestinidad. (Ejemplos son las trayectorias de de Manuel Fernando Contreras o Patricio Hales ${ }^{17}$ ).

En segundo lugar, la vinculación con la configuración de dos tipos de liderazgos en el PCch, que sólo se evidencian hacia fines de los años 80 y que marcan dos formas de hacer y comprender la política. El primero de esos tipos y que mantiene poder real y efectivo en la estructura interna partidaria, corresponde al cuadro clandestino, que perteneciente a la comisión política de la colectividad, realizaba viajes al exterior y se conectaba con la cúpula en el exilio. Su militancia clandestina era relevante para la mantención de la seguridad interna de la colectividad y por ende, de la sobrevivencia no sólo del militante sino que del proyecto político en su conjunto. Este militante, poderoso dentro del partido, era escasamente conocido y se relacionaba muy poco con otros militantes políticos, pero su figura era relevante en la cultura política partidaria.

El segundo de estos tipos, es un militante cuya participación política se va haciendo cada vez más pública en un nuevo escenario abierto con las jornadas de protesta social y la configuración de alianzas políticas cuya actividad fue más o menos tolerada por la dictadura militar. Estos líderes que emergen en organizaciones gremiales, sindicales, universitarias, secundarias u otras, no tienen gran poder al interior de la colectividad, pero van tejiendo redes sociales de conexión con otros liderazgos políticos similares en las otras colectividades de oposición, ampliando su capital social sin que eso se tradujera en incidencia directa dentro de la estructura partidaria. En su mayoría ejerciendo como voceros, vieron limitada su incorporación a la elite partidaria y muchos de ellos, rompieron con la colectividad hacia fines de los 80 , cuando los mundos de la vida dispares generen formas de percibir y enunciar la realidad, definitivamente antagónicas. 


\section{La nueva izquierda: selección y reproducción}

La nueva izquierda está compuesta por aquellas colectividades que nacieron en los años 60 o en 1971, producto de quiebres del centro político o de la izquierda tradicional. Para este estudio corresponde al MIR, al MAPU y a la IC, cuyas fundaciones se dan entre 1965 y 1971.

Una de las principales características de esta nueva izquierda es su marcado carácter generacional (MUÑOZ, 2012). Compuesto mayoritariamente por jóvenes, impregnan a la militancia una identidad caracterizada por cierto mesianismo, estoicismo y una forma de vivir la política muy intensa y hasta desgarradora (MOYANO, 2009).

En general sus liderazgos presentan las siguientes características:

- Provienen de trayectorias militantes de otras colectividades, en algunos casos pertenecían a las cúpulas de sus organismos juveniles, o tempranamente habían sido incorporados a los comités centrales/juntas nacionales partidarias.

- En muchos de los casos provienen de familias vinculadas a la actividad política, ya sea de parentescos directos (padres) o de indirectos (tíos, abuelos, primos, etc.)

- El mayor lugar de politización de estos jóvenes proviene del mundo escolar o universitario, de manera que tienen una vinculación bastante intelectual con la actividad política.

- Mantienen redes, familiares o sociales, con miembros de la elites de otras colectividades, especialmente el mundo demócrata cristiano o del mundo socialista. En esa perspectiva uno de los líderes reclutadores más significativos en esta nueva izquierda es el propio Clodomiro Almeyda.

- Son rápidamente incorporados a una actividad política militante, cuyo principal centro de accionar son organismos de producción pensamiento vinculados a las universidades o directamente al aparato del Estado.

- Asumen actividades de liderazgos muy tempranamente, con un promedio de edad que no supera los 30 años.

Respecto del proceso de selección de estos liderazgos pre 1973, se puede afirmar que dada la estructura relativamente nueva de dichos conglomerados existe un rápido ascenso en la estructura 
Elites de izquierda: experiencias de vida y relatos autobiográficos...

militante, en parte, porque ellos mismo son los fundadores efectivos de estas nuevas colectividades. Lo significativo en este proceso, es que una de las principales fuentes de legitimidad política la constituirá el grupo fundador de la colectividad, quien será depositario y flujo de la institucionalización de nuevos liderazgos.

La mayoría de los militantes de estas colectividades realizan actividades en diferentes frentes de masas con el fin de ir adquiriendo validación entre sus pares. Sin embargo, tanto en el MAPU como en la IC la participación temprana en la Unidad Popular les fue entregando una mayor amplitud de las redes con los otros partidos de la izquierda, a diferencia del MIR cuyo paso a una "semi clandestinidad" los fue aislando de las relaciones socio políticas con el resto de la izquierda, sumado además a las crecientes diferencias ideológicas.

\section{Y cuando se derrumba la democracia.}

Si bien anteriormente hicimos una caracterización de la nueva izquierda en su conjunto, el golpe de Estado imprime diferencias sustanciales a las formas en las que las colectividades reestructuran sus liderazgos partidarios tanto al interior como al exterior del país.

En el caso del MIR, su política del "MIR no se asila" le costó la desaparición de su cúpula política fundadora, quedando sólo pocos miembros del mismo grupo, principales depositarios de la legitimidad instauradora y regeneradora de nuevos líderes. Dado que después del "episodio de Malloco", la dirección se asila en Cuba, la mayoría de los nuevos líderes deben ser investidos por dicha dirección política para realizar trabajo al interior del país.

Ese proceso fue teniendo algunas tensiones al abrirse en los años 80 la posibilidad de una apertura política, lo que terminó desgarrando al MIR hacia los años 1986-1987 en el que un sector, después de una dura evaluación de los propios errores de la colectividad frente a la dictadura, se divide en varias fracciones. El surgimiento de liderazgos nuevos, más partidarios de una práctica política abierta y pública, fueron tensionando al grupo fundador habituado a una práctica de resistencia clandestina y con posturas militares respecto de la derrota a la dictadura. 
De esta forma en el MIR la validación por la vía de la legitimación de parte de los fundadores se pone fuertemente en entredicho hacia mediados de los años 80 y no es capaz de resistirla como colectividad.

En el caso del caso del MAPU y la Izquierda Cristiana es posible afirmar que no siendo víctimas de una gran represión política, sus principales líderes políticos se refugian en el exilio y desde allí ejercen el rol de cooptadores políticos de nuevos liderazgos instaurando un proceso de reproducción e integración sin grandes conflictos internos.

Lo interesante de estos grupos es el uso que hacen para su integración a las elites políticas de la izquierda, del conjunto de contactos que estructuran sus redes sociales, articulando un poderoso capital social tanto hacia el mundo socialista como hacia el mundo demócrata cristiano.

\section{Densidad de Redes entre la vieja y la nueva izquierda: algunos componentes estructurales}

En perspectiva estructural podemos afirmar las siguientes percepciones, que emergen del estudio de las trayectorias biográficas. 
Elites de izquierda: experiencias de vida y relatos autobiográficos...

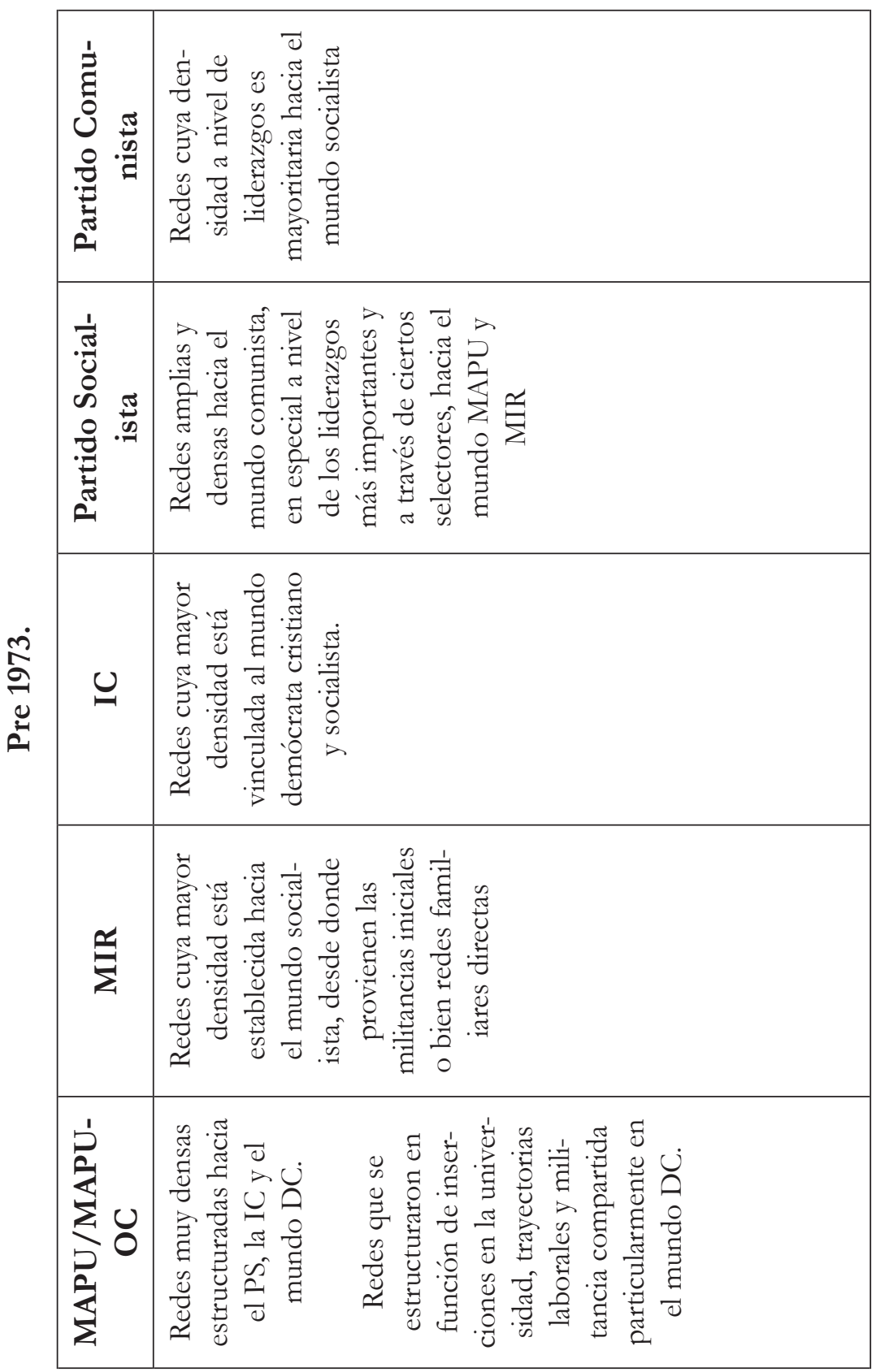

Anos 90, Porto Alegre, v. 20, n. 38, p. 385-410, dez. 2013 


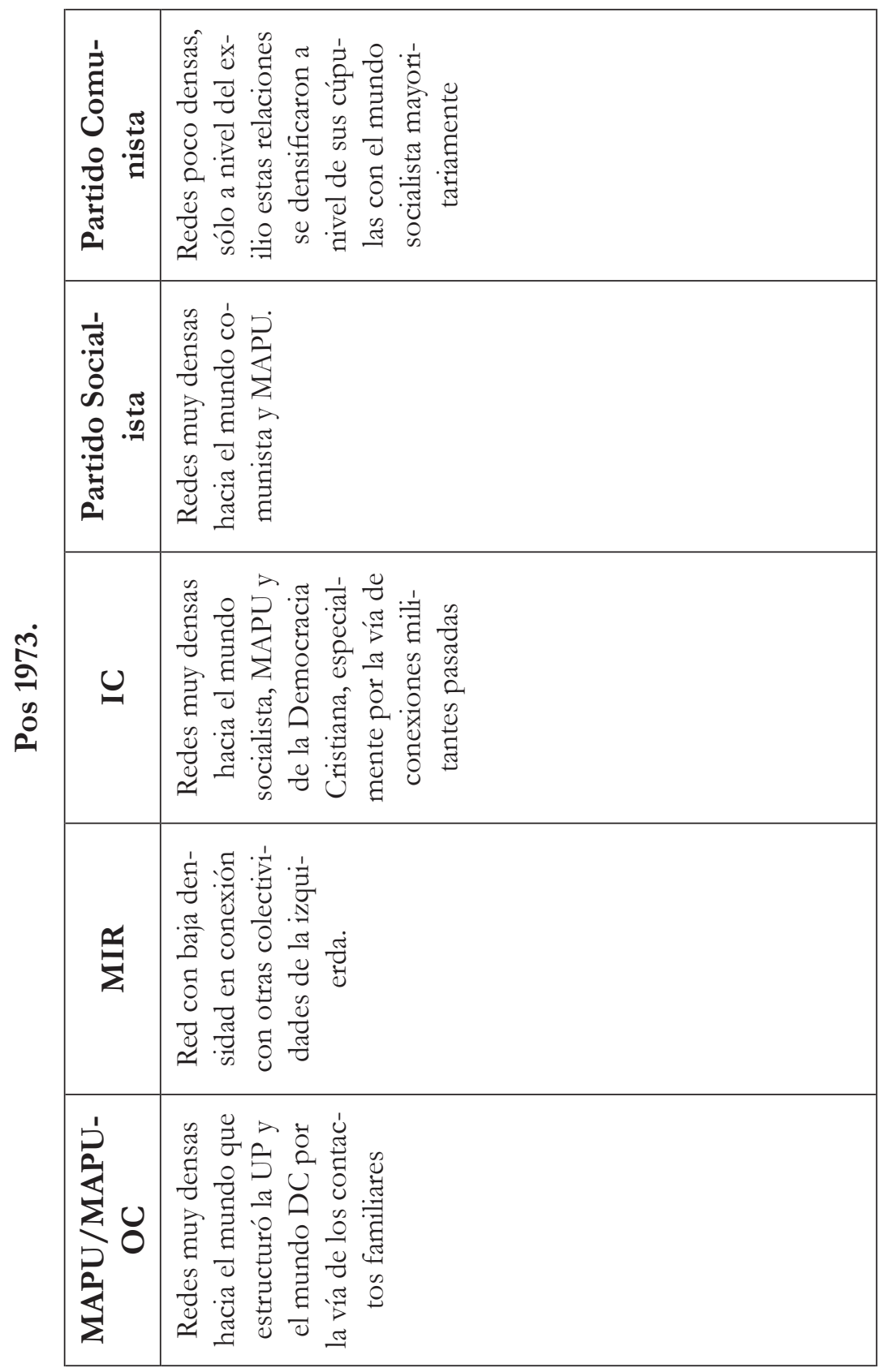


Elites de izquierda: experiencias de vida y relatos autobiográficos...

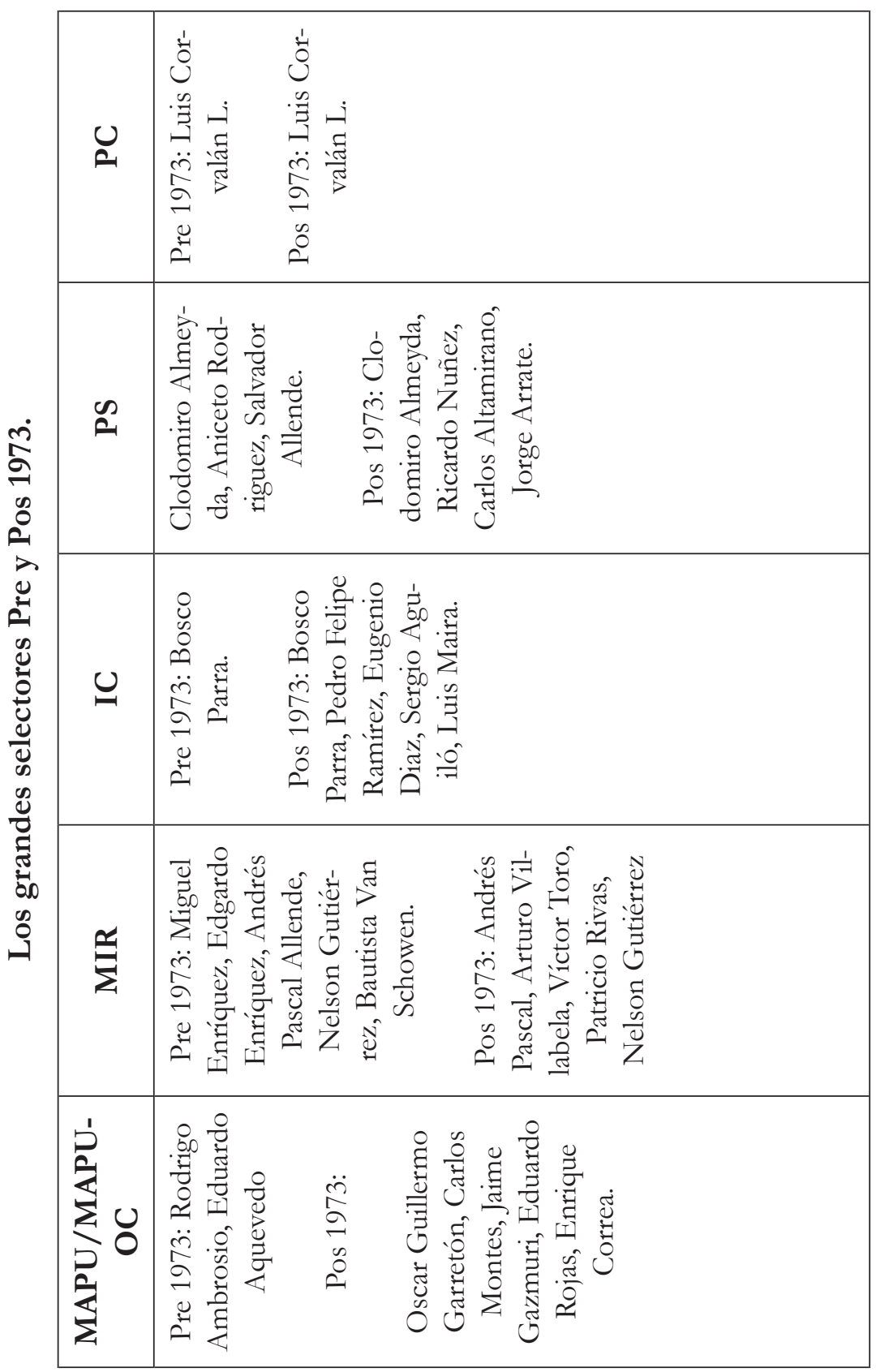

Anos 90, Porto Alegre, v. 20, n. 38, p. 385-410, dez. 2013 
Cristina Andrea Moyano Barahona
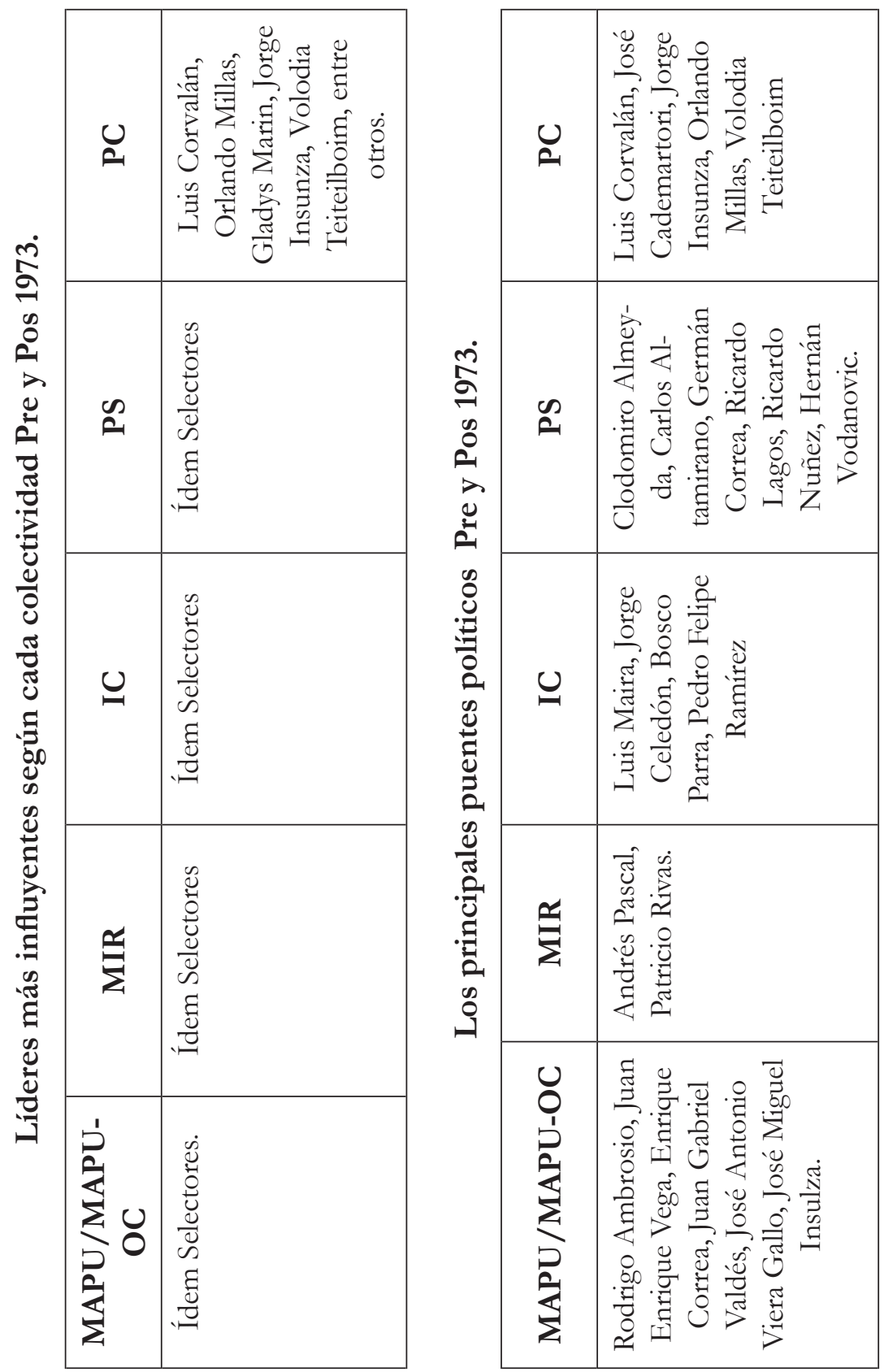

$\stackrel{0}{\bigcirc}$

Anos 90, Porto Alegre, v. 20, n. 38, p. 385-410, dez. 2013 
Elites de izquierda: experiencias de vida y relatos autobiográficos...

De esta forma podemos concluir que el proceso instalado con la dictadura militar consolidó y validó un proceso de reproducción de la elite política basado más en la cooptación y en la cercanía a ciertos líderes, que una práctica política basada en la influencia alcanzada producto de una militancia social y pública.

Pertenecer a una familia política, haber estudiado en determinados colegios, haber sido ayudante o subordinado de algún líder político, contribuyó a generar lazos claves para que una vez que la política entrara en una época de oscuridad y silenciamiento, se convirtieran en los principales ejes sobre los cuales se reprodujo y seleccionó a los miembros de la elite política. Así sin grandes trayectorias política pasadas (pre 1973), un importante grupo de jóvenes se incorporaron a la política, pero a través de una práctica cuya competitividad es menor a las épocas de mayores niveles de actividad pública y democracia.

De allí que la elite de la izquierda gestada durante la dictadura sea muy compacta y con poca capacidad de autogenerarse en un nuevo contexto de mayor apertura política. La urgencia democrática cristalizó un patrón de comportamiento que tiende al anquilosamiento y distanciamiento de la práctica política pública y de masas, tanto de la representación ciudadana como de la construcción de trayectorias políticas vinculadas al mundo social.

En suma, cuando en el marco de la elección del 2005 se afirmaba que estaríamos en presencia de un recambio de las elites, el conjunto de resultados que ha arrojado esta investigación, nos permite concluir que dicha afirmación estuvo muy lejos de la realidad histórica. La elite política de la izquierda se configuró, consolidó y cristalizó, en su estructura más profunda, durante los años de la dictadura, en puente con la vieja elite que vivenció abruptamente el golpe y logró sobrevivir. Esa elite no ha cambiado, los mismos nombres de ahora nos aparecen en las múltiples entrevistas y fuentes documentales consultadas. En suma no ha existido un recambio significativo y para eso hay que entender los procesos históricos que estuvieron detrás de su configuración. 


\section{ELITES OF LEFT SIDE: EXPERIENCES OF LIFE AND AUTOBIOGRAPHICAL STATEMENTS TO UNDERSTAND THE TRANSITION IN CHILE}

Abstract: Article that approaches the configuration, reproduction and mantención of the Chilean elites of left side during the dictatorship, putting special emphasis in the approach of networks and especially, in the analysis of the biographical paths of a set of leaders, in order to understand not only the practices of the above mentioned group, but also the configuration of a political culture that will be key to analyze the process of transition to the democracy in Chile.

Keywords: Elites of left side. Social networks. Autobiographical politicallly active paths.

\section{Notas}

${ }^{1}$ Rango: corresponde al número de lazos de un actor, que puede actuar como receptor de los mismos o como generador.

${ }^{2}$ Grado de intermediación: determina el grado en que una persona actúa como puente o conector entre grupos que de otra forma se mantendrían aislados.

${ }^{3}$ Cercanía: determina cuan cercana está una persona de la otra y si las relaciones son recíprocas o no. La importancia de la conexión de una persona radica en la cercanía respecto de los influyentes en un estudio.

${ }^{4}$ Densidad: corresponde a la cantidad de lazos existentes entre un actor y otro y el número de lazos posibles, dependiendo además si estos son recíprocos o no. Del análisis de la densidad se pueden obtener datos que nos permita pesquisar la existencia de camarillas o subgrupos en los que un actor tiene relaciones más densas.

${ }^{5}$ Jorge Insunza: militante actual del Partido Comunista. Miembro del Comité Central.

${ }^{6}$ Economista y militante de destacada trayectoria al interior del Partido Comunista.

${ }^{7}$ Ingeniero, militante comunista y miembro actual de la Comisión Política del PC.

${ }^{8}$ Ex militante comunista, sociólogo.

${ }^{9}$ Ex Presidente de Chile.

${ }^{10}$ Intelectual y militante socialista, clave en el proceso de la renovación política de dicho sector. Trabajó en Flacso.

${ }^{11}$ Ex militante socialista, ex ministro de Estado de diversas administraciones Concertacionistas y ex candidato a Presidente de la República en la elección del 2010-. 
Elites de izquierda: experiencias de vida y relatos autobiográficos...

${ }^{12}$ Militante socialista, ex ministro de los gobiernos de la Concertación.

${ }^{13}$ Militante socialista, fue miembro del Tribunal Constitucional.

${ }^{14}$ Militante socialista y actual senador de la República.

${ }^{15}$ Militante socialista, ex ministro de administraciones Concertacionistas.

${ }^{16}$ Sobre el tema de la experiencia generacional ver Arostegui y Koselleck.

${ }^{17}$ Actual diputado de la República por el PPD.

\section{Fuentes testimoniales (Escritos personales autobiográficos y entrevistas en profundidad)}

\section{Entrevistas en Profundidad}

Luis Corvalán Lepe.

Manuel Fernando Contreras.

Lautaro Carmona.

Jorge Insunza.

José Cademartori.

Patricio Palma Cousiño.

Patricio Hales.

Carlos Cano.

Pedro Felipe Ramírez.

Sergio Bitar.

Eugenio Tironi.

Carlos Catalán.

José Miguel Insulza.

Ernesto Galaz.

Guillermo Del Valle.

Eduardo Aquevedo.

Juan Gabriel Valdés.

Victor Barrueto.

Juan Enrique Vega.

Mario Alburquerque

Tomás Moulián.

Ricardo Nuñez. 
Ricardo Solari.

Germán Correa.

Hernán Vodanovic.

Jorge Arrate.

Andrés Pascal.

\section{Fuentes testimoniales}

Ismael Llona.

Jaime Gazmuri.

Patricio Rivas.

Carmen Castillo.

Orlando Millas.

Luis Corvalán.

\section{Referencias}

ALVAREZ, Rolando. Arriba los pobres del mundo. Cultura e identidad política del Partido Comunista de Chile entre democracia y dictadura. 1965-1990. Lom ediciones, Chile, 2011. Disponível em: <http://www.ucm.es/info/pecar/Articulos/Villasante21.pdf>. Acesso em: 12 de marzo del 2012.

AROSTEGUI, Julio. La Historia vivida. Sobre la historia del presente. Ed. Alianza, Madrid, 2004. Disponível em: <http://www.ucm.es/info/pecar/Articulos/ Villasante21.pdf>. Acesso em: 12 de marzo del 2012.

KOSELLECK, Reinhart. Los Estratos del Tiempo. Estudios sobre la historia. Ed. Paidós, Barcelona, 2001. Disponível em: <http://www.ucm.es/info/pecar/Articulos/Villasante21.pdf>. Acesso em: 12 de marzo del 2012.

MOYANO, Cristina. "Los líderes de la izquierda. Configuración de las elites en el imaginario político dictatorial chileno y el rol de las revistas políticas de oposición, 1973-1989". En Revista Bicentenerio, Revista de Historia de Cbile y América. Vol 8. No1, 2009. Disponível em: <http://www.ucm.es/info/pecar/Articulos/ Villasante21.pdf>. Acesso em: 12 de marzo del 2012.

MOYANO, Cristina. "Memorias de militantes políticos en Chile e Historia del Presente". En BRESCIANO, Juan Andrés (compilador). El tiempo presente como 
Elites de izquierda: experiencias de vida y relatos autobiográficos...

campo historiográfico. Ensayos teóricos y estudios de casos. Ediciones Cruz del Sur, Uruguay, 2010. Disponível em: <http://www.ucm.es/info/pecar/Articulos/ Villasante21.pdf>. Acesso em: 12 de marzo del 2012.

MOYANO, Cristina. Mapu o la seducción del poder y la juventud. Los años fundacionales del partido mito de nuestra transición, 1969-1973. Ed. Alberto Hurtado, Santiago, 2009. Disponível em: <http://www.ucm.es/info/pecar/Articulos/ Villasante21.pdf>. Acesso em: 12 de marzo del 2012.

MUNOZ, Victor. Generaciones: juventud universitaria e izquierdas políticas en Chile y México. (Universidad de Chile- UNAM, 1984-2006). Lom Ediciones, Santiago, 2012. Disponível em: <http://www.ucm.es/info/pecar/Articulos/ Villasante21.pdf >. Acesso em: 12 de marzo del 2012.

VILLASANTE, Tomás. Redes y sociopraxis. Cuatro redes para vivir mejor. Disponível em: <http://www.ucm.es/info/pecar/Articulos/Villasante21.pdf>. Acesso em: 12 de marzo del 2012.

Recebido em: 28 de agosto de 2012. Aprovado em: 23 de maio de 2013. 